\title{
SPATIOTEMPORAL ANALYSIS OF VARIATIONS OF CERTAIN MORPHOLOGICAL BEACH FORMS
}

\author{
Zbigniew Pruszak $^{1}$, Jan Schönhofer ${ }^{1}$ and Grzegorz Różyński ${ }^{1}$
}

\begin{abstract}
The study is focused on spatiotemporal shoreline variability at a beach with a wide surf zone featuring 3-5 bars based on field observations done between 1983-2008 in the south Baltic Sea. The implementation of various analyses from simple geometry, through spectral analysis up to Discrete Wavelet Transform (DWT) allowed for a synergistic description of simultaneous shoreline and dune foot variability in space and time. The results include meso-scale and long-term phenomena with time scales from several months to many years and spatial scales ranking between a few hundred meters up to several kilometers.
\end{abstract}

Keywords: shoreline, dune foot, non-tidal sea, Baltic Sea, wavelet transform.

\section{INTRODUCTION}

A coastal zone with multiple bars forms a complex morphological system, which is often described by its cross-shore profile. It usually includes the bars themselves as well as the shoreline, emerged beach and dunes. The cross-shore profile characterizes spatiotemporal evolution of the beach system in shore-normal direction, whereas the key indicator of alongshore dynamics is the shoreline together with the associated beach and dune forms.

The intensity and diversity of coastal morphological changes is driven by multiple forces that are random and vary in time and space. Among them the most prominent role is played by waves and the resulting nearshore currents. Due to high complexity of physical processes in coastal zones, their randomness and multitude of the related relationships between them and beach/seabed response, their most realistic assessment can be achieved through in-situ observations and experiments. For this reason, the key objective of the presented research is the analysis and assessment of variability of two major alongshore morphological elements of emerged beach, i.e. the shoreline and the dune system. The dunes and shoreline form an integrated large-scale morphological beach system. In case of sandy beaches the evolution of dunes is usually linked to simultaneous change of shoreline configuration. Thus, studies on natural, undisturbed beaches require a correct range of spatiotemporal scales in order to properly identify geometric characteristics and physical properties of a studied beach. For narrow, intensively eroded beaches, the shoreline and dune foot variations are practically the same. When a wide beach is present the movements of shoreline and dune foot are more or less correlated but never the same; in extreme cases these movements can be completely independent (Pruszak, Schönhofer and Skaja 2008). Such situations are typical for short periods. When the time of observations increases it encapsulates the growing number of extreme hydrodynamic events (storms) and shoreline - dune foot relationships become more likely to emerge. One can also expect a wider envelope of measurements that incorporates the rising range of variability of the studied morphological beach forms. Obviously, the range of shoreline variations is usually greater than that of dune foot. It is related to the fact that the beach response to an extreme event differs from its reaction to normal conditions and that extreme events have a certain probability of occurrence. This probability increases with the time scale of observations. Naturally, the dynamics of beach morphology is also influenced by the bars. They have a decisive role in wave energy dissipation and thus control the amount of energy released on the beach. The correlation analysis between the shoreline and longshore bars, done previously at the study area, found only one significant correlation $R_{I}=0.72$ for the innermost bar. For the offshore bars the associated correlation coefficients are much lower that are equal to $R_{I I}=0.3$ for the $2^{\text {nd }}, R_{I I I}=-0.29$ for the $3^{\text {rd }}$ and $R_{I V}=0$ for the $4^{\text {th }}$ bar. It may imply that only the inner bars $\left(R_{I}, R_{I I}\right)$ exhibit some positive relationship with on- and offshore shoreline movements. The offshore bars show little or no correlations of their positions with respect to the shoreline (Pruszak et al. 1997). Since cross-shore dynamics of the study area, including the shoreline - bars interactions, was presented and discussed in previous contributions, see (Pruszak et al. 1997), (Pruszak et al. 1998), (Pruszak et al. 2008b), the focus of the current study is concentrated on alongshore coastal morphological behavior.

\footnotetext{
${ }^{1}$ Institute of Hydro-Engineering of the Polish Academy of Sciences (IBW PAN) Kościerska 7, 80-328 Gdańsk, Poland
} 
The analysis presented herein includes phenomena on the brink of medium and large scales. The temporal domain covers the periods from seasons to several decades for the spatial coverage of several hundreds of meters up to several kilometers. The major dataset was collected for the period $1983-$ 2008 at IBW PAN Coastal Research Station (CRS) at Lubiatowo, PL, situated at south Baltic Sea coast. The analysis is a continuation of the previous piece of research, (Pruszak, Schönhofer and Skaja 2008). Its novel element, expanding the past contributions, is a synergistic implementation of various methods of analysis starting with the basic geometric considerations, through spectral analysis up to the state-ofthe-art discrete wavelet transform. Additionally, another novelty lies in attempt of simultaneous scrutiny of spatial and temporal variability of large alongshore morphologic features.

\section{STUDY AREA, CONDITIONS AND SCOPE OF RESEARCH}

The beach in the vicinity of CRS Lubiatowo is featured by a mildly sloping $(\beta=0.015)$ cross-shore profile with multiple bars, varying beach width and moderately pronounced berm, see Fig. 1 . Three to five permanent bars together with an ephemeral, frequently disappearing bar in close shoreline proximity can be distinguished. The ephemeral bar strongly interacts with the shoreline; it is washed out together with the receding beach during storms and contributes to beach recovery throughout mild hydrodynamic regimes. The average sediment diameter in the submerged part of the beach oscillates about $D_{50}=0.022 \mathrm{~cm}$.

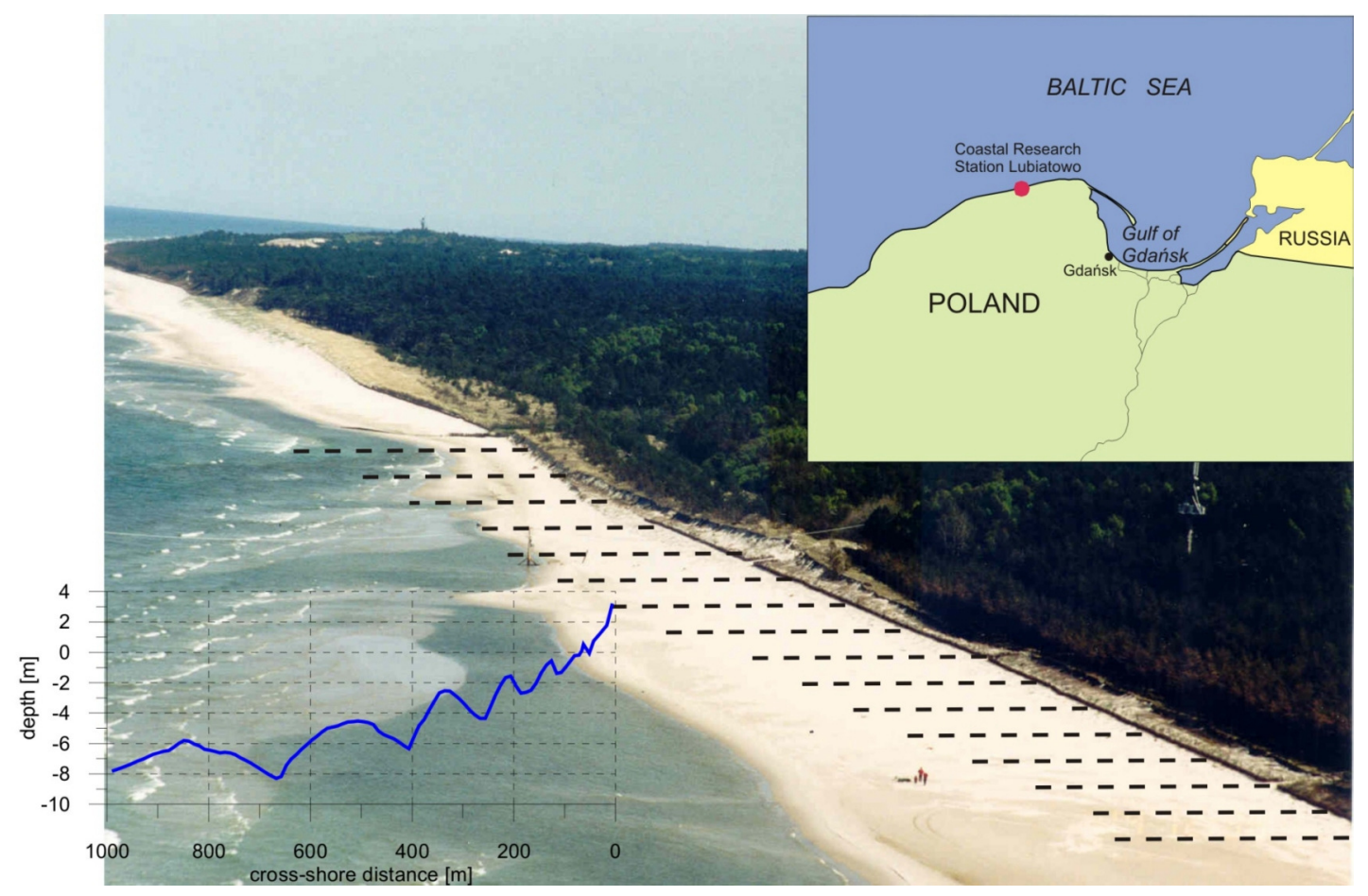

Figure 1. Study site.

The incoming deepwater waves are first transformed in the surf zone and then its energy is dissipated there. The rate of energy dissipation at beaches with multiple bars depends on several main factors. Among them deepwater wave parameters play an important role. Equally vital are cross-shore beach characteristics, particularly the number of bars that largely contribute to wave breaking intensity. Small and moderate deepwater waves release energy nearer the shore; whereas high, stormy waves lose energy gradually on the consecutive bars due to multiple breaking. Thus, most energy of large waves is dissipated on greater depths, over the offshore bars and only a small fraction of their initial energy can reach the shoreline. Since smaller waves cannot be broken over the offshore bars, their loss of energy is practically negligible and the shoreline must absorb it all. The measurements, carried out at the study area, demonstrated that if deepwater wave height $H_{r m s}$ exceeds $1.5 \mathrm{~m}$, the average energy loss over the offshore bars $I I$, III and $I V$ is more than $80 \%$ of the initial, deepwater wave energy. If the deepwater 
wave height $H_{r m s}$ oscillates about $0.5 \mathrm{~m}$ the average rate of dissipation is roughly $60 \%$. It means that in such instances only $40 \%$ of the initial, deepwater wave energy affects the shoreline, (Pruszak et al. 2008c).

The records of yearly wave climate at CRS Lubiatowo, done in 2007-2008, found that the mean annual wave energy $\bar{E}_{0}$ at a depth $h=15 \mathrm{~m}$ amounts to some $0.88 \cdot 10^{5} \mathrm{~J} / \mathrm{m}$, with the max/min values of $3.4 \cdot 10^{5} \mathrm{~J} / \mathrm{m}$ and $0.1 \cdot 10^{5} \mathrm{~J} / \mathrm{m}$ respectively. Due to seasonality the spring - summer energy is equal to some $0.46 \cdot 10^{5} \mathrm{~J} / \mathrm{m}$ vs. $1.33 \cdot 10^{5} \mathrm{~J} / \mathrm{m}$ in autumn - winter. Knowing that for average waves some $60 \%$ of deepwater wave energy is dissipated over the bars it can be assumed that near the shoreline, that is at depths $h \approx 0.5 \mathrm{~m}$, the energy flux is in the range of $\left(\bar{E}_{0}\right)_{h \approx 0,5 m}=0.4 \cdot 0,88 \cdot 10^{5} \approx 0.37 \cdot 10^{5}[\mathrm{~J} / \mathrm{m}]$, (Pruszak et al. 2008c). Consequently, due to oblique wave approach, only a fraction of the energy flux $E_{0}$ affects the shoreline directly and remodels it. This reasoning highlights the role of offshore bars that dissipate most of the incoming deepwater wave energy and act as a natural beach protection. Apart from the wave action other external forces, encountered in the surf zone, can contribute to shoreline/seabed changes. They include various currents and water flows. Among them the most prominent is the wavedriven longshore current; near CRS Lubiatowo it can reach over $1 \mathrm{~m} / \mathrm{s}$; during heavy storms it can even approach the value of $V \approx 1.5 \mathrm{~m} / \mathrm{s}$.

The observations indicate that during storms the shoreline retreat can reach up to $0.7 \mathrm{~m} / \mathrm{day}$. For extreme events the retreat can exceed $1 \mathrm{~m} /$ day easily. Beach recovery after storms occurs at a considerably lower pace of about not more than $0.4 \mathrm{~m} /$ day. The critical value of energy flux below/above which the shoreline advance/retreat can be expected can be defined as $\left(\vec{E}_{0}\right) k r y t \approx 5 \cdot 10^{4}[\mathrm{~J} / \mathrm{m}]$. The only exception can occur in winter when the ice barrier enhances resilience of the beach against erosion (Pruszak, Ostrowski, Szmytkiewicz and Schönhofer 2009).

The analyzed coastal segment covers the distance of some $2.7 \mathrm{~km}$. The measurements were carried out on a monthly basis along 27 cross-shore profiles, evenly spaced every $\Delta x=100 \mathrm{~m}$. Thus, each record of shoreline/dune foot positions consists of $\mathrm{M}=27$ numbers. These measurements are jointly plotted in Fig. 2; they cover the period of 1983-2008. The horizontal axis is compatible with local geodesic enumeration for the whole 27 cross-shore profiles. Hence, the alongshore locations of shoreline $\left(y_{b}\right)$ and dune foot $\left(y_{w}\right)$ variations with respect to the local geodetic base, are defined by the numbers $(i)$.

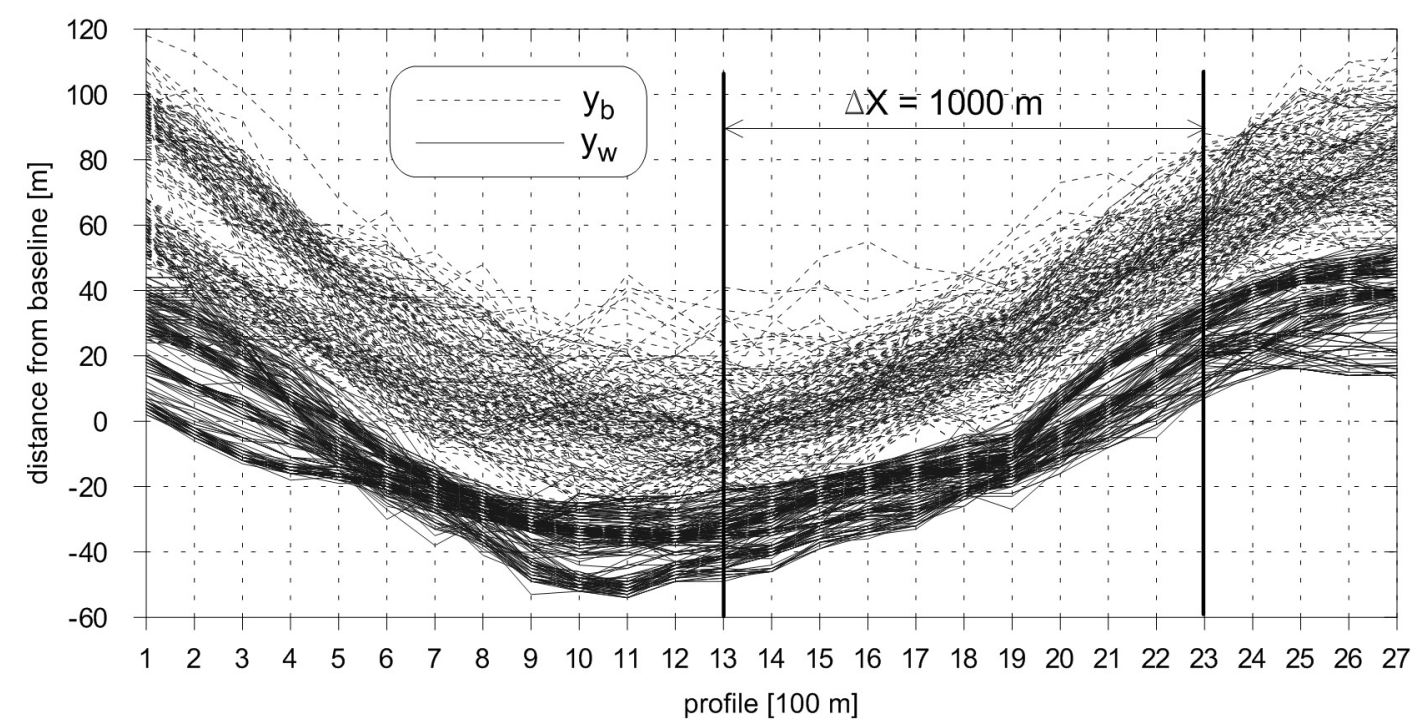

Figure 2. Collective chart of recorded shoreline $\left(y_{b}\right)$ and dune foot $\left(y_{w}\right)$ configurations. 


\section{EVOLUTION (OSCILLATIONS) OF SELECTED ALONGSHORE MORPHOLOGICAL FORMS. GEOMETRIC AND STOCHASTIC ANALYSIS OF SHORELINE AND DUNE FOOT MIGRATION}

\section{Geometric method}

Let us adopt the description of shoreline and dune foot variations according to a schematic shown in Fig. 3. Then, let us assume that each change of position of the shoreline points (i) by a distance $\Delta y$, defined by two consecutive measurements, can be described by actual and absolute values $\Delta y_{i}$ and $\left|\Delta y_{i}\right|$ respectively. The actual displacements $\Delta y_{i}$ describe real migrations of the points $i(i=1,2 \ldots 27)$ of the lines $y_{b}$ or $y_{w}$, whereas the absolute ones $\left|\Delta y_{i}\right|$ map their dynamics between two measurements $(k+1)$ and $(k)$ irrespective of the direction of change.

$$
\begin{gathered}
\Delta y_{i}=\left(\Delta y_{i}\right)_{k+1}-\left(\Delta y_{i}\right)_{k} \\
\left|\Delta y_{i}\right|=\left(\left|\Delta y_{i}\right|\right)_{k+1}-\left(\left|\Delta y_{i}\right|\right)_{k}
\end{gathered}
$$

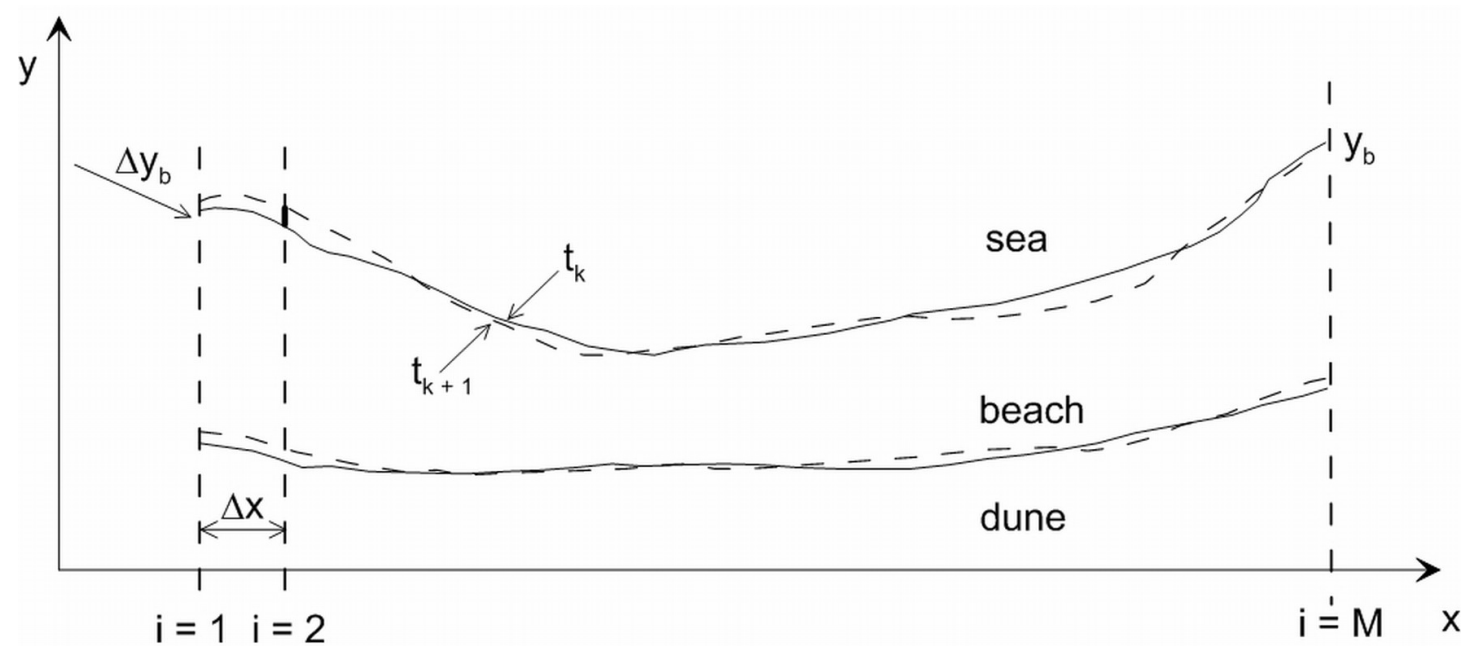

Figure 3. Definition sketch.

When all measurements of each point $(i)$ are taken into account we obtain the summed final displacements of the lines $y_{b}$ or $y_{w}$ after the time $\Delta t=2008-1983 \approx 25$ yrs. For the actual displacements they are given by the sums $y_{i}=\sum_{1}^{N}\left(\Delta y_{i}\right)_{k}$ that define the final position of $y_{b}$ or $y_{w}$ of each point $(i)$. The final absolute displacements $\left|y_{i}\right|=\sum_{1}^{N}\left|\left(\Delta y_{i}\right)_{k}\right|$ yield in turn the distance that each point $(i)$ traveled over 25 years of measurements. The index $k=1, \ldots, N$ represents the number of measurements of lines $y_{b}$ or $y_{w}$ over the time $\Delta t$; it was in the range of $N \approx 190$. Graphical representations of shoreline and dune foot movements for the period $\Delta t=2008-1983$ are plotted in Fig. 4 (actual displacements) and Fig.5 (absolute displacements). 

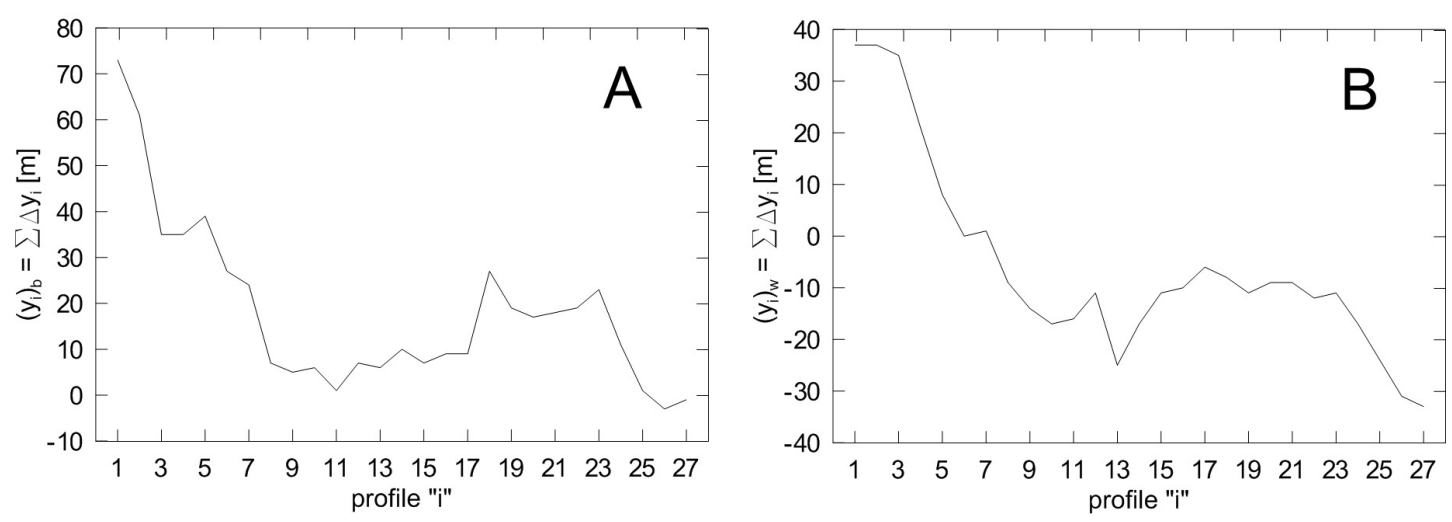

Figure 4. Summed actual shoreline (A) and dune foot (B) displacements between $1983-2008$
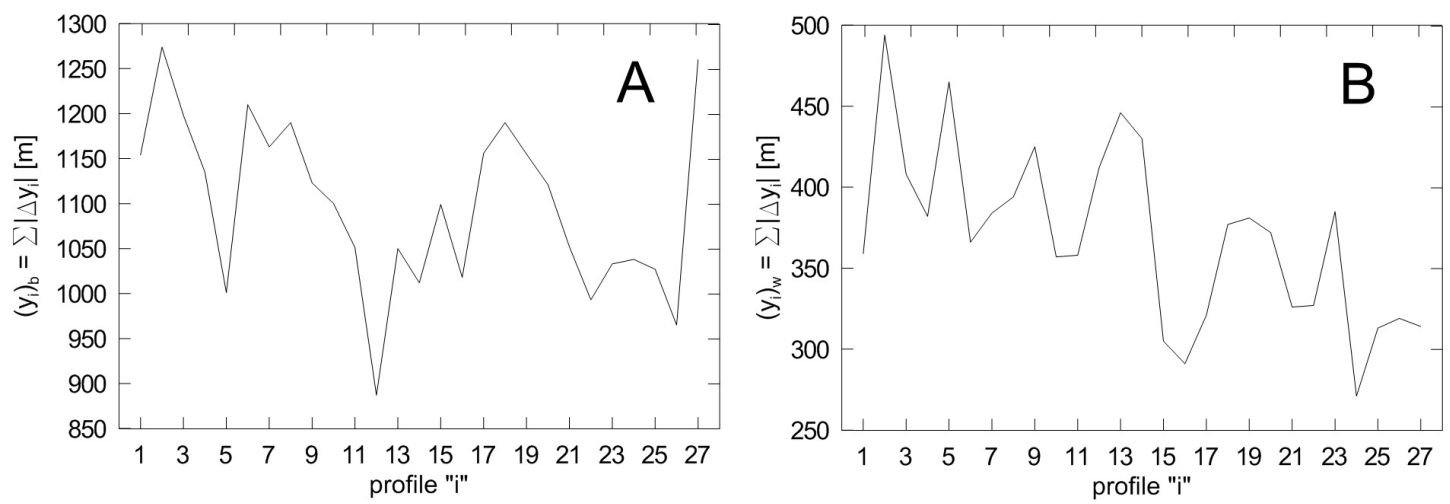

Figure 5. Summed absolute shoreline (A) and dune foot (B) displacements between 1983 - 2008

These plots represent the resultant cross-shore migration of shoreline $y_{b}$ and dune foot $y_{w}$ positions from 1983 till 2008. Obviously, apart from spatial variations, beach morphology is also subject to time scale dependent variability. The time scale related evolution can be studied for very short periods, e.g. duration of a single storm (hours to days), medium scales of one to several years and very long scales featuring decadal and centennial changes. From the engineering point of view short and medium scales are of key focus.

The relationship between medium time scales and the magnitude and character of morphological beach changes can be traced in Fig. 6 . The longer the time scale (expressed by $N$ ) the more hydrological situations were incorporated and the more divergent trajectories of all analyzed points became. The growing divergence demonstrates a large degree of independent evolution originating from significant morphodynamic differentiation of alongshore coastal processes even at short coastal segments. 


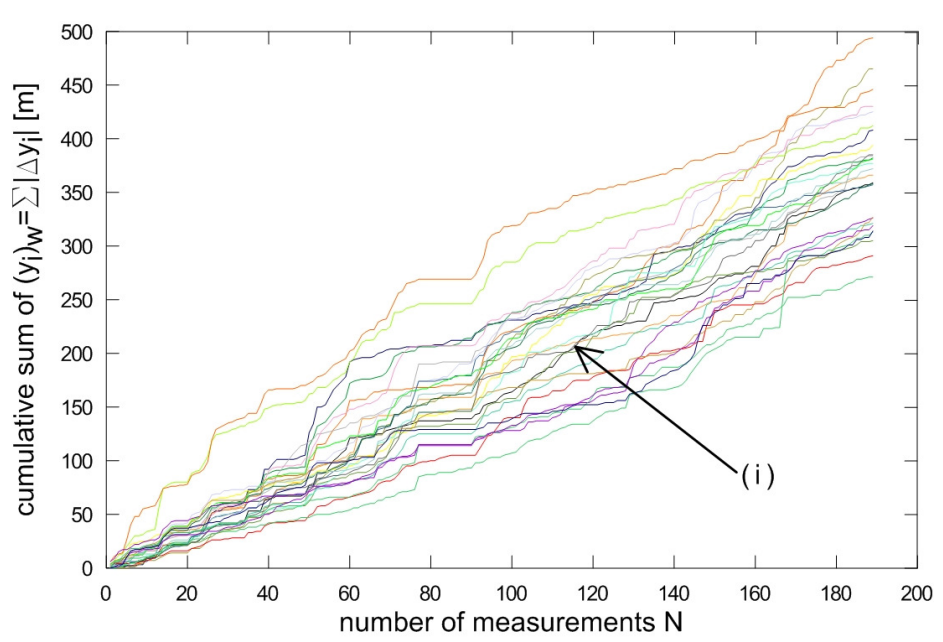

Figure 6. Summed absolute dune foot displacements as function of measurements $N$

Further geometric analysis of alongshore morphological forms in medium time scales was executed for shoreline positions, because this quantity is much more dynamic than the dune foot. The adopted time scale was equal to the time interval $\Delta t_{s}=1996-1999$, situated in the middle of the entire data registration period.

For the clarity of the analysis let us introduce the quantities $S_{o}$ and $S_{w}$, describing alongshore temporal shoreline variability. The quantity (3) represents the area between two consecutive shoreline measurements; this area takes an active role in shoreline evolution no matter if it erodes or accretes. The parameter (4) in turn describes the resultant depletion or accretion of beach surface between two consecutive shoreline measurements.

$$
\begin{aligned}
& S_{O}=\sum_{1}^{M}\left|P_{i}\right| \\
& S_{W}=\sum_{1}^{M} P_{i}
\end{aligned}
$$

In these formulas $P_{i}$ denotes a field between two profile lines of the neighboring points $(i)$ between two consecutive measurements.

The quantities (3) and (4) are 2D generalizations and extensions of the previously defined linear (1D) parameters (1) and (2). The negative values of $P_{i}$, means the erosion of a segment between the points $i$ and $(i+1)$; when they are positive this segment undergoes accumulation.

The variations of $S_{o}$ and $S_{w}$ was related to mean monthly deepwater wave energy $\bar{E}_{0}$ and its shore normal flux $\left(\bar{E}_{0}\right)_{n} \quad \bar{E}_{0}$ characterizes deepwater wave energy between two shoreline measurements; it can be described by equation (5):

$$
\bar{E}_{0}=\sum_{i}^{n} E_{k} / n=\sum_{1}^{n}\left[(1 / 8 n)\left(\rho g H_{k} L\right)\right.
$$

The number $n$ represents individual wave energies $E_{k}$, corresponding to wave heights $H_{k}$, between two consecutive shoreline measurements. Fig. 7A, B demonstrates temporal variations of $S_{o}$ and $S_{w}$ and energy parameters $\bar{E}_{0}$ and $\left(\bar{E}_{0}\right)_{n}$.

It may be a bit surprising that the relations between deepwater wave energy and the variations of $S_{o}$ and $S_{w}$ are not particularly high. However, more detailed scrutiny shows that they do exist and are more pronounced than the first glance assessment tells. It can be attributed to complex, multidimensional shoreline responses, frequently obscuring the effects of incoming wave energy fluxes. Good examples are Nov. 1997 or Apr. and Nov. 1998, when for rather low energetic conditions both parameters were 
large and almost equal, that is $S_{o} \approx+S_{w}$. This equality means that all shoreline changes had the same sign, which in physical terms means substantial and persistent accumulation. Inverse situations occurred during high energy events, such as in Dec. 1996, Sep. 1997 or Feb. 1999, when the beach responded by vivid erosion and both parameters were roughly equal with the opposite sign $S_{o} \approx-S_{w}$. Further analysis of Fig. 7 reveals that for above average energy inputs both parameters vary considerably, that is $S_{o}>>$ $S_{w}$, see e.g. Dec. 1997 or Aug, 1998. Physically, it can be interpreted as multidimensional shoreline oscillations without a clear trend.

In sum, when the ration $S_{w} / S_{o} \approx \pm 1$ the beach exhibits either a clear erosion (-) or accretion (+), in the case $S_{w} / S_{o} \approx 0$ the beach stays in relative equilibrium, when smaller, local oscillations are possible. Finally, for $0<S_{w} / S_{o}<1$ the beach undergoes intermediate stages. It should be added that some untypical random events can produce difficulties as to the clear interpretation of variation tendencies of the parameters $S_{o}$ and $S_{w}$.

The geometric assessment of shoreline variations indicates it may contain some periodic components. Identification of such periodic components and their trends requires relevant statistical and stochastic tools. Thus, classical spectral analysis, using the Fast Fourier Transform (FFT) algorithm was applied parallel to state-of-the-art Discrete Wavelet Transform (DWT); the selection of two methods was aimed at precise determination of periodic components from morphological beach data.
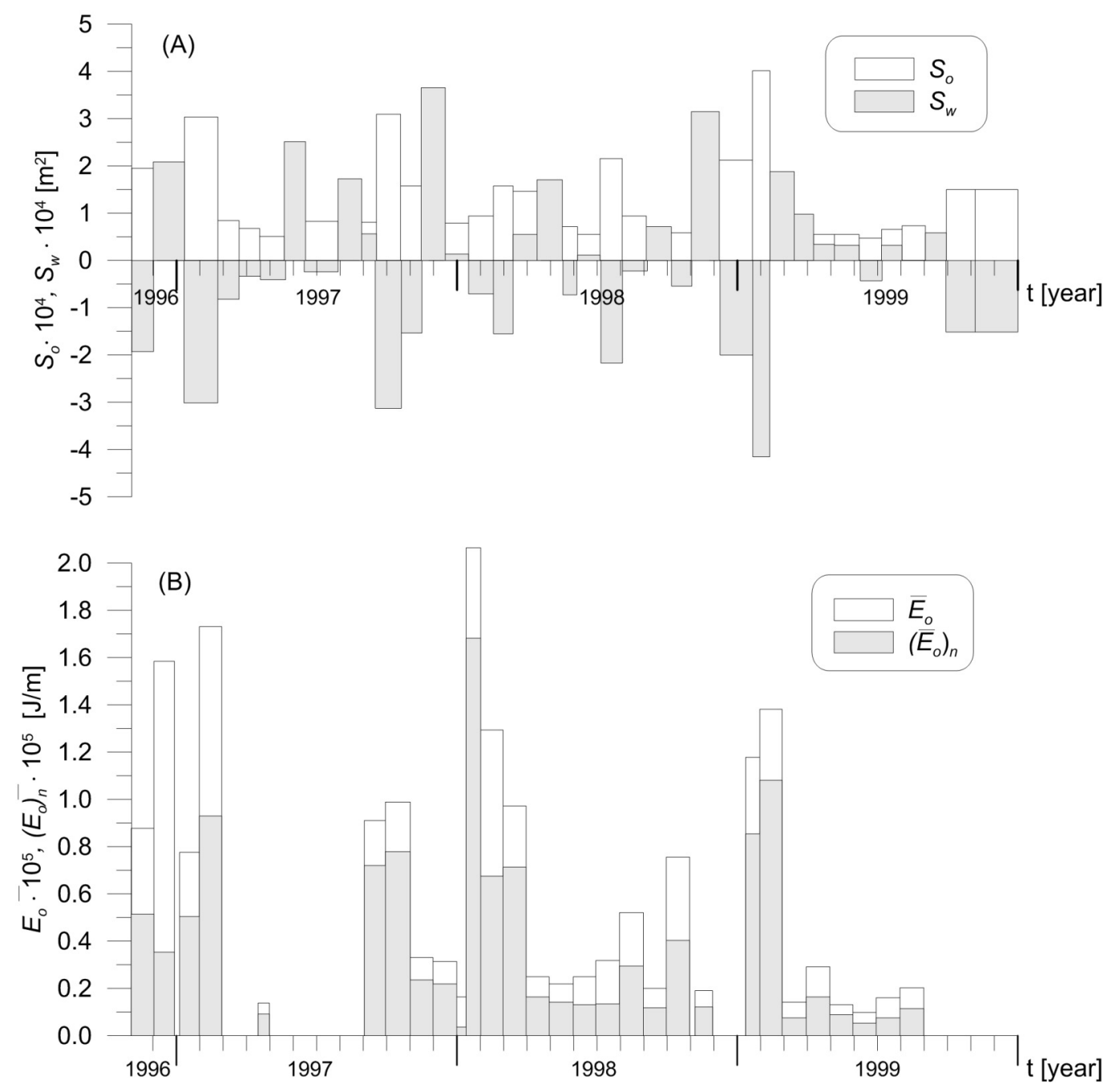

Figure 7. Shoreline evolution in terms of parameters $S_{o}$ and $S_{w}(\mathrm{~A})$ and total deepwater wave energy $\bar{E}_{0}$ and its shore-normal flux $\left(\bar{E}_{0}\right)_{n}$ (B). 


\section{FFT - Fast Fourier Transform}

As is commonly known the FFT analysis resolves a studied signal into periodic components and ranks them as to the amount of signal variance they carry. Therefore, it is well suited for identification of time scales that are characteristic for spatiotemporal shoreline evolution of a naturally evolving beach. Assuming that shoreline measurements can be interpreted as stochastic processes, the associated spectral density functions $S(\omega)$ can be calculated using equation (6):

$$
S(\omega)=2 \int_{-\infty}^{+\infty} F(\tau) \exp (-2 \pi j \omega \tau) d r
$$

In this equation $F(\tau)=1 / T \int_{0}^{T} x(t) \cdot x(t+\tau) d t$ is the autocorrelation function, $\tau$ the time lag in the measured signal $(t)$ and $\omega-$ is the frequency. The signal can be reconstructed using formula (7):

$$
x(t)=x_{o}+\sum_{k=1}^{\infty} A_{k} \cos \left(2 \pi k \omega_{1} t-\theta_{k}\right)
$$

where: $A_{k}$, and $\theta_{k}$,-are the $k$-th amplitude and phase of harmonic Fourier series components and $\omega_{l}$ is the frequency of the $1^{\text {st }}$ harmonic component.

Two profiles were selected for the analysis, separated by the alongshore distance $\Delta X=1 \mathrm{~km}$ (points 13 and 23 in Fig. 2). Spectral density functions $S(\omega)$ were computed for these points, also key features of their trajectories $x(t)$ were reconstructed with the most significant Fourier coefficients. The points that were selected for the Fourier transform represent different spatial phases of shoreline evolution. Exemplary details of FFT analysis - spectral density $S(\omega)$ and the reconstruction of basic signal features at point 23 are plotted in Fig. 8. 

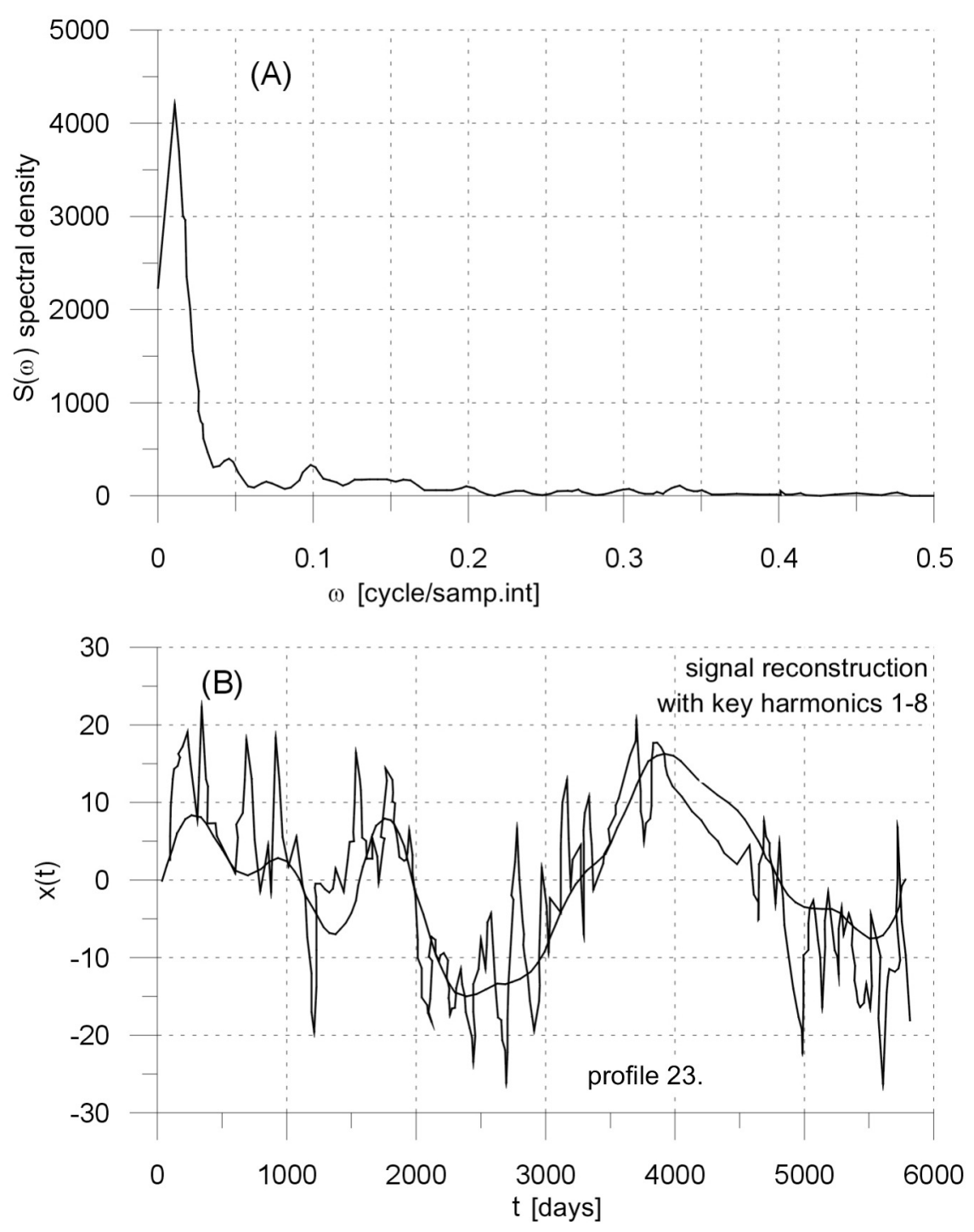

Figure 8. Shoreline variability at point 23 in terms of FFT: spectral density (A), signal reconstruction with key harmonics 1 - $8(B)$

The comparison of dominant harmonic components from two locations (points 13 and 23) reveals a significant correlation $R>0.5$. Spectral density in turn provides information of the most significant periodic characteristics. For point 23 it is roughly equal to 8 year, whereas for point 13 it is considerably greater than the study period. It can only be hypothesized that this period is in the range of 20 - 30 years. Further periodic modes are much less significant; although a mode incorporating seasonal shoreline migration is discernible. The results in general confirm the fact that the studied shoreline can have different dominant periodic modes at relatively short longshore distances. It confirms both temporal and spatial variability of shoreline rhythmic behavior. Also, the results are consistent with past study results with multi-channel singular spectrum analysis (Różyński 2005).

\section{DWT- Discrete Wavelet Transform}

DWT is one of the recent and very effective methods of processing even short and noisy signals. Unlike classical spectral techniques it does require the assumption of signal stationarity. Thus, it is also very well suited for extraction of information from signals generated by beaches.

A wavelet function can be defined as: 


$$
\psi_{\lambda, t}(u)=\frac{1}{\sqrt{\lambda}} \psi\left(\frac{u-t}{\lambda}\right)
$$

In equation (8) $\lambda$ represents scale and $t$ location (or time). Changing the scale $\lambda$ the function $\psi(u)$ can be dilated when $\lambda>1$ or contracted for $\lambda<1$, the change of $t$ corresponds to translation along time. Each function has a unit energy for all $\lambda$ and $t$ :

$$
\int_{-\infty}^{\infty}\left|\psi_{\lambda, t}\left(\frac{u-t}{\lambda}\right)\right|^{2} d u=1
$$

Apart from these properties wavelet functions have other features, such as rapid decay for $u$ far from $t$ and zero mean value:

$$
\int_{-\infty}^{\infty} \psi_{\lambda, t}\left(\frac{u-t}{\lambda}\right) d u=0
$$

Zero mean value and rapid decay indicate wavelets are single wave forms with unit energy ensured by the scaling factor $1 / \sqrt{\lambda}$.

Discrete wavelet representation can be obtained in the following manner. Let us assume $\lambda=\lambda_{0}^{m}$, where $m$ is integer and $\lambda_{0}>1$ is an a priori adopted scale. Time $t$ can be defined as $t=n t_{0} \lambda_{0}^{m}$, where $n$ is integer and $t_{0}>0$ depends on a particular wavelet function. Now the wavelet function can be presented in a discrete form as:

$$
\psi_{m, n}(u)=\frac{1}{\sqrt{\lambda_{0}^{m}}} \psi\left(\frac{u-n t_{0} \lambda_{0}^{m}}{\lambda_{0}^{m}}\right)=\lambda^{-m / 2} \psi\left(\lambda_{0}^{-m} u-n t_{0}\right)
$$

For such a discrete wavelet function we can now determine the discrete wavelet transformation of a signal $f(t)$ :

$$
W f(m, n)=\lambda^{-m / 2} \int_{-\infty}^{\infty} f(u) \psi\left(\lambda_{0}^{-m} u-n t_{0}\right) d u
$$

The most convenient discrete representation of any wavelet function $\psi_{m, n}(u)$ is obtained for $\lambda_{0}=2$ and $t_{0}=1$ :

$$
\psi_{m, n}(u)=2^{-m / 2} \psi\left(2^{-m} u-n\right)=\frac{1}{\sqrt{2^{m}}} \psi\left(\frac{u-2^{m} n}{2^{m}}\right)
$$

Also, the interpretation of wavelet analysis is the easiest and frequently most insightful when their set $\left\{\psi_{m, n}\right\}$ forms an orthonormal basis for all $m$ and $n$ (Mallat 1989). Then all finite signals $\left(\int_{-\infty}^{\infty} f^{2}(t) d t<\infty\right)$ can be approximated with arbitrary precision by the linear combination of $\psi_{m, n}$ :

$$
f(t)=\sum_{m=-\infty}^{\infty} \sum_{n=-\infty}^{\infty} D_{m, n} \psi_{m, n}
$$

In this expression the first (external) sum is done with respect to scales, from small to large ones, and for each scale an internal summation is done on translations. The coefficients $D_{m, n}$ are calculated as integrals of the product of the studied signal and wavelet, for a given scale and translation: 


$$
D_{m, n}=\int_{-\infty}^{\infty} f(u) \psi_{m, n}(u) d u
$$

Thus, $D_{m, n}$ is a measure of information about the signal $f(t)$ for the scale $2^{m}$ and translation (time) $2^{m} n$; the coefficients $D_{m, n}$ vary in time.

The most advanced application of wavelets is related to multi-resolution analysis; it allows breaking down the analyzed signal into features having different resolutions. Let us denote with $f_{m}(t)$ the approximation of the processed signal $f(t)$ every $2^{m}$-th point. With the growth of $m$ the scale decreases, but the resolution increases; the quantity $f_{m}(t)$ is called smooth representation of the signal for the scale $m$. The next level of resolution consists in addition of the so called detail $f_{m}^{\prime}(t)$ :

$$
f_{m-1}(t)=f_{m}(t)+f_{m}^{\prime}(t)
$$

The smooth representation $f_{m}(t)$ is obtained by means of the expansion using the 'smoothing functions', whereas the detail $f_{m}^{\prime}(t)$ with the expansion using the wavelet functions. Thus, the smooth representation $f_{m}(t)$ is evaluated with the following sum (Kumar et al. 1997):

$$
f_{m}(t)=\sum_{n=-\infty}^{\infty} C_{m, n} \phi_{m, n}(t)
$$

where:

$$
\phi_{m, n}(t)=2^{-m / 2} \phi\left(2^{-m} t-n\right)
$$

is the smoothing function. The coefficients $C_{m, n}=\int_{-\infty}^{\infty} \phi_{m, n}(u) f(u) d u$ allow for determination of discrete values of the signal $f(t)$ for the scale $m$ and translation $n$, so the scaling function $\phi(t)$ works like the sampling function. The detail $f_{m}^{\prime}(t)$ is in turn calculated with discrete wavelet functions as the sum:

$$
f_{m}^{\prime}(t)=\sum_{n=-\infty}^{\infty} D_{m, n} \psi_{m, n}
$$

for $D_{m, n}$ obtained from equation (8). Importantly, the choice of the scaling function $\phi(t)$ results in automatic selection of the wavelet function $\psi(t)$ and vice versa, so both functions form a definite pair.

The multiresolution analysis of morphodynamic data from Lubiatowo was done using a nearly symmetric coif5 wavelet function. It is the smoothest function available in standard wavelet toolboxes.

Short-term variability of morphodynamic data, usually related to a single storm and very dynamic, was filtered out prior to the analysis. Then, using the wavelet transforms for the analysis of shoreline and dune foot variability, it was identified that they both exhibit similar long-term evolution pattern. It has a form of a standing wave; its length can be estimated as $L \approx 3,700 \mathrm{~m}$ (Fig. 9) and the period as $T \approx 30$ years (Fig. 10).

The standing wave has maximum amplitude of $\pm 15 \mathrm{~m}$ for the dune foot and $\pm 30 \mathrm{~m}$ for shoreline. Obviously, other smaller and greater spatial undulations are superimposed on this dominant feature, but they remain constant in time, so are of much less interest. At the most, these undulations can describe characteristic beach configurations that remain stable for more than 20 years now. Since the long-term spatial shoreline and dune foot variability is characterized by the standing wave, its amplitude varies in the alongshore direction. It can be easily observed in Fig. 11, where three profile lines ( $i=3,15$ and 23), situated far and near the node are shown. 

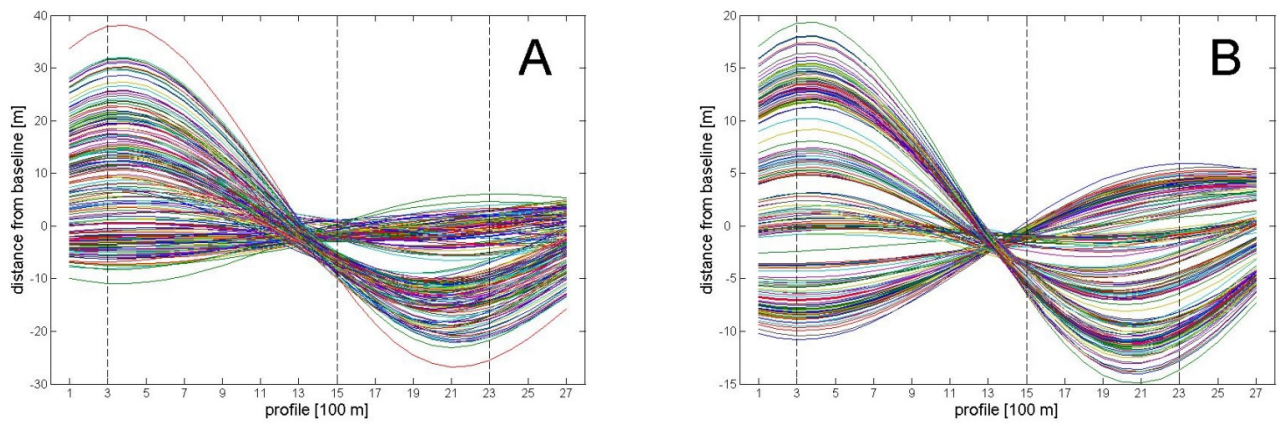

Figure 9. Spatial variation of shoreline (A) and dune foot (B)
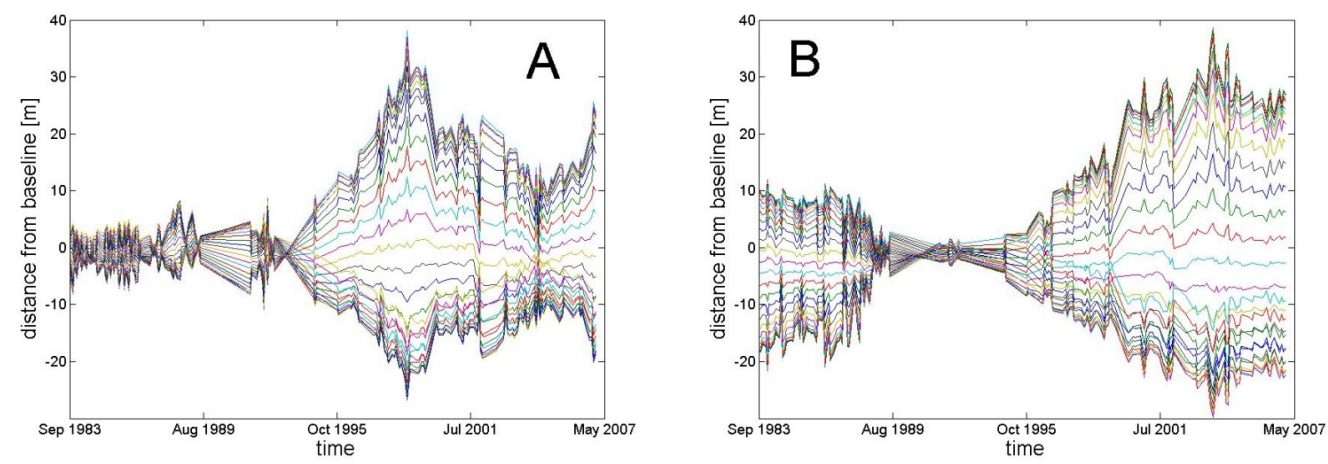

Figure 10. Temporal variation of shoreline (A) and dune foot (B)

The complexity and multitude of possible shoreline and dune foot spatiotemporal oscillations are reflected by their 3D graphical representation in Fig. 12. This graph clearly shows how complicated these changes are and how many scales they contain. Obviously, the scales have different weights and thus bearing, so the description of shoreline and dune foot morphodynamic variability is even more complicated.

It should be emphasized that one of the merits of application of DWT was the extraction of spatiotemporal patterns, which are demonstrated in Fig. 12. Apart from illustrating very complex 3D character of shoreline and dune foot variations, this graph also demonstrates that various spatial and temporal scales can be related to each other; this conclusion is contradictory to common assumptions, encountered in coastal science that they are (to a large extent) independent.

\section{DISCUSSION AND CONCLUSIONS}

As a result of multiple breaking over multiple bars, only a certain fraction of deepwater wave energy reaches and remodels the shoreline proximity. If the mean wave energy flux is oblique to the shore, the resultant energy input the beach must absorb is additionally diminished. When deepwater significant wave height $H_{s}$ exceeds $1.5 \mathrm{~m}$, more than $80 \%$ of incoming energy is dissipated over multiple bars; thus less than $20 \%$ arrives near the shore.

When $H_{s}<0.5-1.0 \mathrm{~m}$, about $30-50 \%$ of deepwater wave energy can reach the shoreline proximity. This demonstrates predominant role of nearshore bars as (natural) energy dissipaters that prevent enhanced beach erosion.

The action of dynamic and random wave and current fields produces diverse spatiotemporal variability of emerged beach, predominantly the shoreline. Apart from short-term variations related to instantaneous influx of energy to the shore, other phenomena, occurring in longer scales, are present. They can account for seasonal variations of the beach or other seasonal climate changes and also for alongshore migration of beach macro-forms. The majority of such phenomena are related to medium scales. Local, temporary departures from the trends, observed in longer time scales, result to a large degree from random nature's character. Such departures may originate from untypical and hardly 
predictable extreme events or human induced interferences that introduce chaos into the quasi-orderly physical system.
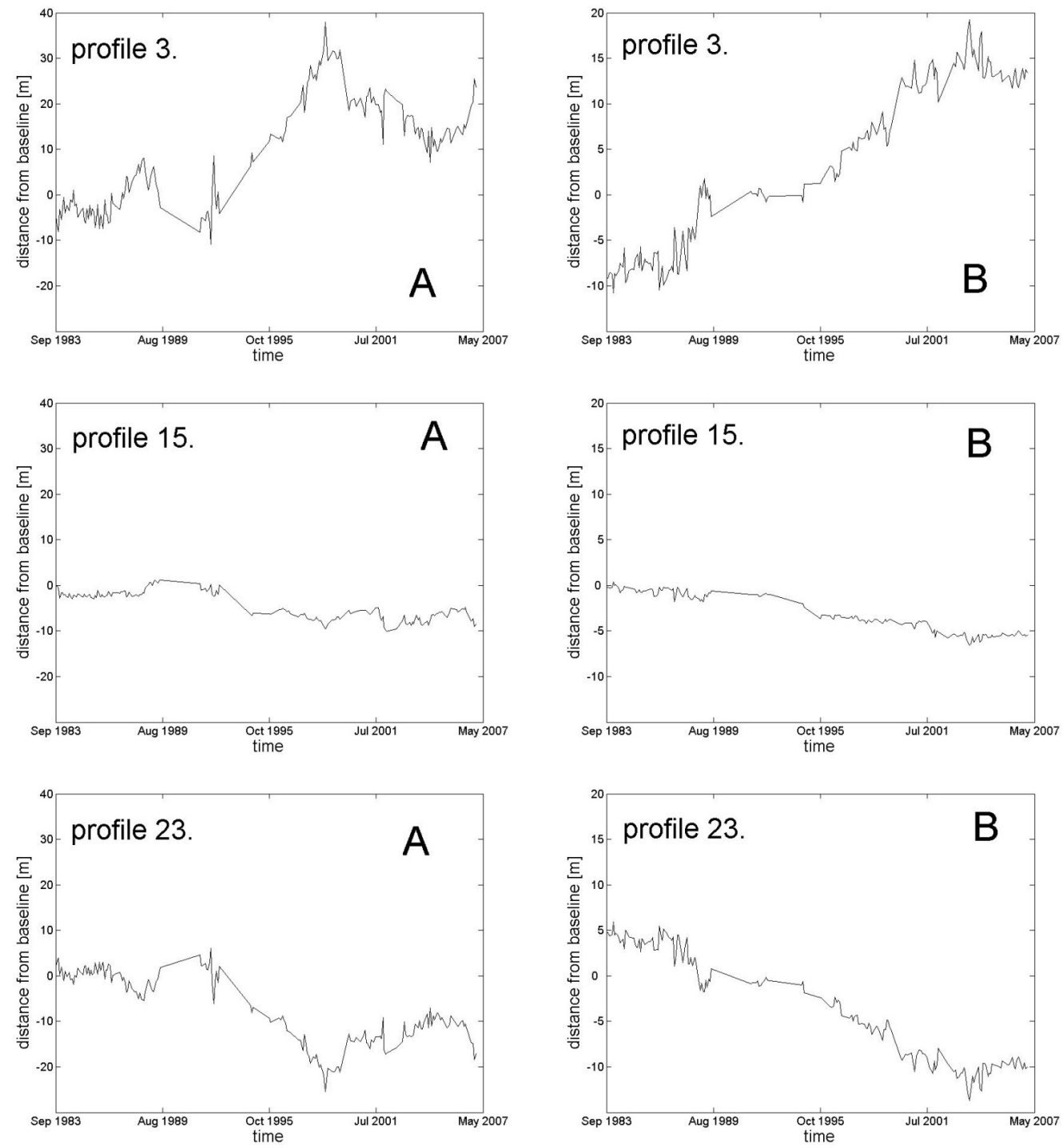

Figure 11. Temporal migration of shoreline (A) and dune foot (B) at profile lines 3,15 and 23

Alongshore shoreline and dune foot oscillations, covering a wide range of scales and obtained with various methods of analysis, prove that very complex mechanisms, producing such variability, are at work. It is particularly visible in shoreline evolution, where sea and land intersect. Therefore, the shoreline is constantly subjected to direct and highly dynamic sea action. As the presented study shows spatiotemporal variations of alongshore forms of beach morphology can appear as progressive or standing waves. A certain analogy to smaller rhythmic beach forms, such as beach cusps or mega-cusps can be noticed here. These forms were also analyzed with spectral tools and DWT, e.g. (Pruszak et al 2007) or (Pruszak et al 2008b). The genesis of those forms was related either to infragravity, particularly edge waves, or to beach self organizing processes. The shoreline and its parameters are very sensitive to various, often untypical disturbances of wave energy supplied near the shore. The joint analysis of long-term energy fluxes and beach parameters $S_{o}$ and $S_{w}$ reveals that not only in winter times but also in summer seasons the shoreline exhibits non-trivial activity. 

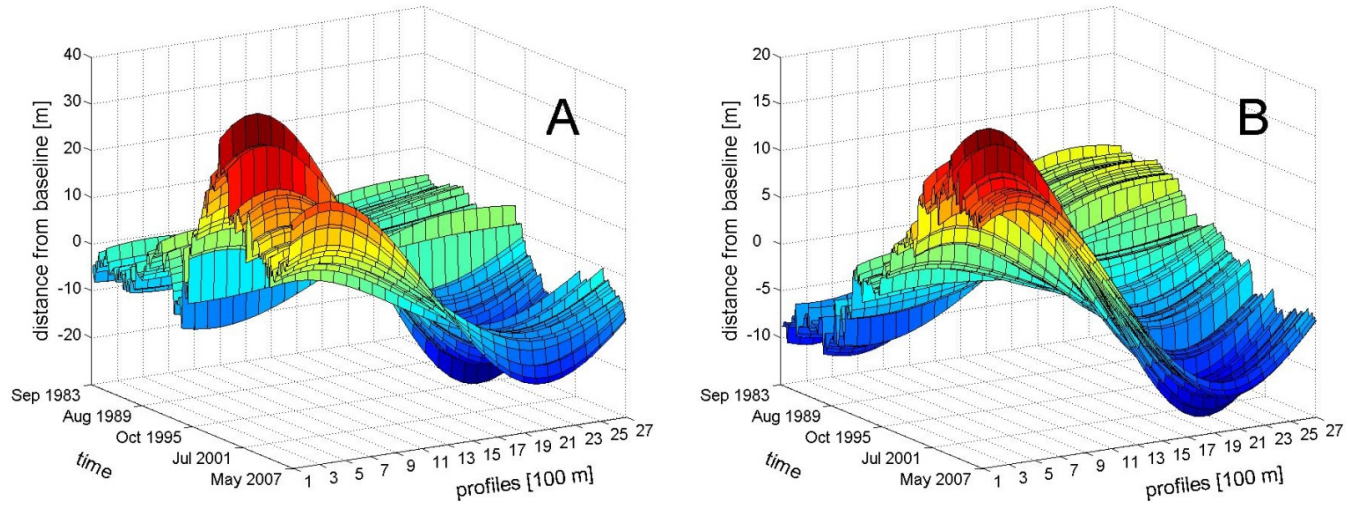

Figure 12. Spatiotemporal shoreline (A) and dune foot (B) variability

Using the FFT method an attempt was made to determine long-term trends of shoreline variability. As a result, a strong periodic component with $T \approx 8$ years was identified. In this case the correlation between shoreline records and reconstruction with key Fourier coefficients was found to be relatively high with $R>0.5$. This periodicity is similar to large scale atmospheric North Atlantic Oscillation, discovered by G. Walker in 1920. Therefore, the 8-years shoreline cycle can be linked to large-scale atmospheric oscillations, generated over the north part of the Atlantic Ocean; this connection suggests that such global atmospheric patterns can contribute to morphodynamic changes on Baltic Sea coasts.

The second harmonic is much less explicit. It has a period of some $20-30$ years and precise determination of that cycle is not possible, because of much shorter period of data used for the analysis. Other periodic components were found to be much less significant, although seasonal shoreline migration was discernible. The results lead to the conclusion that even fairly closely located points of shoreline exhibit different patterns of variability. Therefore, it confirms the fact that both temporal and spatial shoreline rhythmicity do vary substantially.

Using the DWT method separately for shoreline and dune foot records joint spatiotemporal patterns, featuring the complex character of evolution of these lines, were obtained. Importantly, it was found that time and spatial scales are not independent but they are closely related to each other.

DWT patterns contain the dominant large-scale component, whose alongshore wavelength is equal to $L \approx 3700 \mathrm{~m}$ and the related period $T \approx 30$ years, see Fig. 9 . This spatiotemporal wave is the standing wave with maximum amplitude reaching $\pm 15 \mathrm{~m}$ for the dune foot and $\pm 30 \mathrm{~m}$ for shoreline. Naturally, other smaller and greater spatial fluctuations are superimposed on this pattern. However, they show little variation in time, so they are hardly interesting. The complexity and multitude of possible shoreline and dune foot oscillations are proven by the complicated multidimensional distribution that maps the joint spatiotemporal variability of these parameters of alongshore beach morphology.

\section{ACKNOWLEDGMENTS}

The support of the European Commission through FP7-SPACE-2009-1, Contract 242284 FIELD_AC ("Fluxes, Interactions and Environment at the Land-Ocean Boundary. Downscaling, Assimilation and Coupling."), is gratefully acknowledged.

\section{REFERENCES}

Kumar, P. and Foufoula-Georgiou, E. 1997. Wavelet analysis for geophysical applications. Reviews of Geophysics, 35, 4, Nov. 1997, 385-412.

Mallat, S. 1989. A theory for multi-resolution signal decomposition: The wavelet representation. IEEE Trans. Pattern Anal. Mach. Intel., 11(7), 674-693.

Pruszak, Z., Różyński, G. and Zeidler, R. 1997. Statistical proporties of bars of multi-bar profiles. J. Coastal Engineering, Elsevier Science Publishers, 1997, No 31, 263-280.

Pruszak Z. and G. Różyński. 1998. Variability of Multibar Profiles in Terms of Random Sine Functions, J. Waterway, Port, Coastal and Ocean Engineering, ASCE, Vol. 124, No. 2, 48-56. 
Pruszak Z., Różyński G., Szmytkiewicz M. and Skaja M. 2007. Field observation of edge waves and beach cusps on the South Baltic Sea Coast, Journal of Coastal Research, Vol. 23, No 4, 846-860.

Pruszak Z., Schonhofer J. and Skaja M. 2008a. Variability of shoreline and dune on South Baltic coast, Proceedings of the $9^{\text {th }}$ International Conference LITTORAL2008, November 25-28 Venice, 67,1-9.

Pruszak Z., Różyński G. and Szmytkiewicz P. 2008b. Megascale rhythmic shoreline forms at a beach with multiple bars, Oceanologia, 50 (2), 183-203.

Pruszak Z., Szmytkiewicz P, Ostrowski R., Z., Skaja M. and Szmytkiewicz M. 2008c. Shallow-water wave energy dissipation in a multi-bar coastal zone, Oceanologia, 50 (1), 43-58.

Pruszak Z., Ostrowski R., Szmytkiewicz P. and Schönhofer J. 2009. Field studies on wave-induced shallow water morphological features, Proceedings of $31^{\text {rd }}$ International Conference on Coastal Engineering, Hamburg, August/September 2008.

Różyński, G. 2005. Long term shoreline response of a non-tidal, barred coast, Coastal Engineering, 52 , 79-91. 